\title{
ASSOCIATIVE ALGEBRAS SATISFYING A SEMIGROUP IDENTITY
}

\author{
DAVID M. RILEY \\ Department of Mathematics, The University of Alabama, Tuscaloosa, AL 35487-0350, USA \\ e-mail:driley@gp.as.ua.edu \\ MARK C. WILSON \\ Department of Mathematics, University of Auckland, Private Bag 92019 Auckland, New Zealand \\ e-mail:wilson@math.auckland.ac.nz
}

(Received 4 February, 1998)

\begin{abstract}
Denote by $(R, \cdot)$ the multiplicative semigroup of an associative algebra $R$ over an infinite field, and let $(R, \circ)$ represent $R$ when viewed as a semigroup via the circle operation $x \circ y=x+y+x y$. In this paper we characterize the existence of an identity in these semigroups in terms of the Lie structure of $R$. Namely, we prove that the following conditions on $R$ are equivalent: the semigroup $(R, \circ)$ satisfies an identity; the semigroup $(R, \cdot)$ satisfies a reduced identity; and, the associated Lie algebra of $R$ satisfies the Engel condition. When $R$ is finitely generated these conditions are each equivalent to $R$ being upper Lie nilpotent.
\end{abstract}

1991 Mathematics Subject Classification 16R40, 20M07, 20M25

1. Introduction and statement of results. A well-known result due to Levitzki [3] states that every finitely generated bounded nil ring is nilpotent. Not long ago, Zel'manov proved the Lie-theoretic analogue: every finitely generated Lie ring satisfying the bounded Engel condition is nilpotent [19]. The corresponding problem in the category of groups is the famous Burnside problem. The construction by Adian and Novikov of infinite finitely generated groups of finite exponent provided a negative solution to this problem. See [1].

The Burnside problem has some natural generalizations. For example, the problem of whether or not every Engel group is locally nilpotent remains open [17]. Because every nilpotent group is known to satisfy a semigroup identity [5,8], a weaker version of this problem has also been posed: does every Engel group satisfy a semigroup identity [6, Problem 2.82]? Even the following question remains open: can an Engel group contain a free (noncommutative) subsemigroup? See [10]. blems.

Recently, the present authors settled the ring-theoretic analogues of these pro-

Recall that a ring $R$ satisfies the Engel identity of degree $n$ if and only if

$$
e_{n}:=[x, \underbrace{y, y, \ldots, y}_{n}]
$$

is identically zero in $R$; whereas, $R$ is said to be upper Lie nilpotent if the descending central series of associative ideals $\left\{\gamma^{i}(R)\right\}$ in $R$ defined by $\gamma^{1}(R)=R$, $\gamma^{i+1}(R)=\left\langle\left[\gamma^{i}(R), R\right]\right\rangle$ reaches zero in finitely many steps. In addition to the usual multiplicative semigroup, $(R, \cdot), R$ forms a semigroup, denoted by $(R, \circ)$, under the circle operation $x \circ y=x+y+x y$. We proved in [14] that every finitely generated 
associative ring $R$ satisfying the Engel condition is upper Lie nilpotent. From this result we were able to infer that whenever $R$ satisfies an Engel identity then both the associated circle and multiplicative semigroups of $R$ must satisfy a so-called Morse identity.

Define sequences $f_{n}$ and $g_{n}$ by $f_{1}(x, y)=x y, g_{1}(x, y)=y x$, and

$$
\begin{aligned}
& f_{n+1}\left(x, y, x_{3}, \ldots, x_{n+2}\right)=f_{n}\left(x, y, x_{3}, \ldots, x_{n+1}\right) x_{n+2} g_{n}\left(x, y, x_{3}, \ldots, x_{n+1}\right), \\
& g_{n+1}\left(x, y, x_{3}, \ldots, x_{n+2}\right)=g_{n}\left(x, y, x_{3}, \ldots, x_{n+1}\right) x_{n+2} f_{n}\left(x, y, x_{3}, \ldots, x_{n+1}\right),
\end{aligned}
$$

for all $n \geq 1$. The $n$th Mal'cev identity [5] is the semigroup identity

$$
f_{n}\left(x, y, x_{3}, \ldots, x_{n+1}\right)=g_{n}\left(x, y, x_{3}, \ldots, x_{n+1}\right),
$$

while the $n$th Morse identity $u_{n}(x, y)=v_{n}(x, y)$ [7] is the $n$th Mal'cev identity with $x_{3}=\cdots=x_{n+1}=1$.

Consequently, neither $(R, \cdot)$ nor $(R, \circ)$ can contain a free subsemigroup if $R$ satisfies an Engel identity.

The problem of characterizing finitely generated groups satisfying an arbitrary semigroup identity has been studied by several authors (see, for example, [4], [18] and [16]). Because this class of groups contains the Burnside groups, this problem is highly nontrivial, especially in the light of the recent construction by Ol'shanskii and Storozhev of a 2-generated group satisfying a semigroup identity that is not a periodic extension of a locally soluble group [9].

Algebras over fields of characteristic zero which satisfy a circle semigroup law, and a more general semigroup condition called collapsibility, were studied previously by the first author in [12]. In sharp contrast to the combinatorial methods employed in this paper, the techniques used in [12] rely heavily on deep structure theorems from both group and ring theory. In this article we study associative algebras that satisfy an arbitrary semigroup identity. In fact, we obtain a partial converse to our result in [14].

Throughout the remainder of this paper, $K$ will denote an infinite commutative domain and $R$ an associative $K$-algebra on which the action of $K$ is torsion-free; (this occurs, for example, when $K$ is an infinite field). All identical relations in algebraic objects will be assumed to be nontrivial unless otherwise stated. A semigroup $S$ satisfies an identity if and only if there are distinct words $u, v$ in the free semigroup on

$$
X=\left\{x=x_{1}, y=x_{2}, x_{3}, x_{4}, \ldots\right\}
$$

so that $u=v$ in $S$. The semigroup identity is left reduced if the first letters of $u$ and $v$ are different, right reduced if the last letters of $u$ and $v$ are different and simply reduced if it is both left and right reduced. In other words, $u=v$ is reduced if and only if $u v^{-1}$ and $v^{-1} u$ are reduced words in the free group on $X$. If $(R, \cdot)$ (respectively $(R, \circ))$ satisfies an identity we often say that $R$ satisfies a semigroup identity (respectively, a circle semigroup identity). Clearly each of these corresponds to a polynomial identity in $R$. A generalization of a multiplicative semigroup identity in $R$ is a binomial identity, a polynomial identity of the form $\alpha_{1} u_{1}+\alpha_{2} u_{2}=0$, where $u_{1}, u_{2}$ are monomials and $\alpha_{1}, \alpha_{2} \in K$. The various types of reduced binomial identities are defined in the obvious way. 
Tasic and the first author proved in [13] that $R$ is Lie nilpotent of class at most $n$ if and only if $(R, \circ)$ satisfies the $n$th Mal'cev identity. The main result in the present article further demonstrates the close relationship between the Lie structure of $R$ and semigroup properties of $R$.

THeOREM 1.1. Let $R$ be a K-algebra. Then the following statements are equivalent.

(i) $R$ satisfies a circle semigroup identity;

(ii) $R$ satisfies a reduced semigroup identity;

(iii) $R$ satisfies a reduced binomial identity;

(iv) $R$ satisfies an identity of the form $\sum_{i=0}^{n} \alpha_{i} y^{i} x y^{n-i}=0, \alpha_{i} \in K, \alpha_{0} \neq 0, \alpha_{n} \neq 0$;

(v) $R$ satisfies an Engel identity;

(vi) $(R, \circ)$ satisfies a Morse identity.

Furthermore, for any two conditions A, B from (i)-(vi), our proof gives (sometimes theoretical) bounds for the degree of the identity in B in terms of the degree of the identity in A. In particular, these bounds do not depend on $R, K$ or the characteristic of $K$. Notice, too, that since every finite semigroup (in particular $(R, \circ)$, where $R$ is a finite ring) satisfies an identity, some hypothesis on the coefficient ring $K$ is required. The following example demonstrates that the distinction between reduced and arbitrary multiplicative semigroup identities is also necessary.

EXAmple 1.2. Let $R$ be the subalgebra of the matrix algebra $M_{2}(K)$ spanned by the matrix units $e_{11}$ and $e_{12}$. Then $[R, R] \subseteq K e_{12}$, and so $R$ satisfies the semigroup identity $[x, y] z=x y z-y x z=0 . R$ does not satisfy any Engel identity, since $\left[e_{11}, e_{12}\right]=e_{12}$. Thus, by Theorem $1.1, R$ does not satisfy any reduced semigroup identity, nor any circle semigroup identity.

TheOrem 1.3. Let $R$ be a $K$-algebra, where $K=p>0$. Then the following statements are equivalent.

(i) $R$ satisfies a semigroup identity;

(ii) $R$ satisfies a binomial identity;

(iii) $R$ satisfies an identity of the form $\sum_{i=0}^{n} \alpha_{i} y^{i} x y^{n-i}=0, \alpha_{i} \in K$;

(iv) $R$ satisfies an identity of the form $y^{m} e_{m} y^{m}=0$.

We remark that the characteristic zero analogue of Theorem 1.3. is stated in [2]; however, their result corresponding to our implication (iv) $\Rightarrow$ (i) is not proved and does not seem obvious to the present authors.

The fact that $R$ is non-unital is essential to Example 1.2, as indicated by the following proposition.

Proposition 1.4. Let $R$ be a unital K-algebra. If $R$ satisfies a semigroup identity then $R$ satisfies the corresponding reduced semigroup identity.

THEOREM 1.5. There exists a function $f$, depending only on natural numbers $d$ and $n$, such that if a K-algebra $R$ satisfies a circle semigroup identity of degree $n$ and $R$ is generated over $K$ by d elements then $R$ is upper Lie nilpotent of index at most $f(d, n)$. 
2. Semigroup identities. Our hypotheses on $K$ were chosen to imply, by the usual Vandermonde determinant argument, that every homogeneous component of a polynomial identity for $R$ is also a polynomial identity for $R$ (see [11, 6.4.14]). We shall use this key fact freely, without explicit mention.

By a partial linear identity we shall mean an identity of the form

$$
\sum_{i=0}^{n} \alpha_{i} y^{i} x y^{n-i}=0
$$

with $\alpha_{i} \in K$. Such an identity will be called left reduced if $\alpha_{0} \neq 0$, right reduced if $\alpha_{n} \neq 0$ and reduced if it is both left and right reduced.

Proposition 2.1. Let $R$ be a K-algebra.

(i) If a semigroup $S$ satisfies an identity in $x, y, x_{3}, \ldots$ that is left reduced, right reduced or reduced, then $S$ satisfies an identity, of the same type, in $x$ and $y$ only.

(ii) If $R$ satisfies a binomial identity then $R$ is bounded nil or $R$ satisfies a semigroup identity.

(iii) If $R$ satisfies a binomial identity that is left reduced, right reduced or reduced, then $R$ satisfies a partial linear identity of the same type.

(iv) If $R$ satisfies the identity $y^{n}=0$, then $R$ satisfies $e_{2 n-1}=0$.

Proof. Suppose without loss of generality that our left reduced identity has the form

$$
x x_{i_{1}} \cdots x_{i_{m}}=y x_{j_{1}} \cdots x_{j_{n}} .
$$

Recall that we identify $x=x_{1}$ and $y=x_{2}$. Substituting $x_{i}=x y^{i},(i \geq 3)$, we obtain a left reduced identity in $x$ and $y$ only. If the original identity were right reduced as well, then $x_{i_{m}} \neq x_{j_{n}}$. Thus, by an appropriate permutation of the variables, we obtain an equivalent identity of the form

$$
x_{k_{1}} \cdots x_{k_{m}} x=x_{l_{1}} \cdots x_{l_{n}} y .
$$

Substituting $x_{i}=x y^{i},(i \geq 3)$, into this identity and then concatenating on the right with the 2-variable left reduced identity yields the 2-variable reduced identity:

$$
x x_{i_{1}} \cdots x_{i_{m}} x_{k_{1}} \cdots x_{k_{m}} x=y x_{j_{1}} \cdots x_{j_{n}} x_{l_{1}} \cdots x_{l_{n}} y .
$$

This and symmetry prove (i).

Next, given a binomial identity $\alpha_{1} u_{1}+\alpha_{2} u_{2}=0$ holding in $R$, set all variables equal, to $y$ say. If the identity is not homogeneous, then separating components shows that $R$ is bounded nil. On the other hand, if it is homogeneous then $\left(\alpha_{1}+\alpha_{2}\right) y^{n}=0$, for some $n$, so that either $R$ is bounded nil or $\alpha_{1}=-\alpha_{2}$, in which case $u_{1}-u_{2}=0$ holds in $R$. This proves (ii).

In order to prove (iii), suppose that $R$ satisfies a given binomial identity. Observe from (ii) that either $R$ is bounded nil, in which case $R$ satisfies a partial linear identity by (1) in the proof of (iv) below, or $R$ satisfies a semigroup identity, 
which by (i) can be taken to be of the form $u(x, y)-v(x, y)=0$. Thus we may assume that $R$ is not bounded nil, and hence that the semigroup identity is homogeneous. We assert that the homogeneous component of degree 1 in $x$ of the identity $u(x+y, y)-v(x+y, y)=0$ is a partial linear identity. To see why it is nontrivial, write $u=a u^{\prime}, v=a v^{\prime}$, where $a$ has length $m$ and $u^{\prime}=v^{\prime}$ is a left reduced equation. If (as we may assume without loss of generality) $u^{\prime}$ starts with $x$ and $v^{\prime}$ with $y$, then in the expansion of $u(x+y, y)$ there is precisely one monomial starting with $y^{m} x$, whereas no monomial in the expansion of $v(x+y, y)$ begins with $y^{m} x$. This, and symmetry, yields (iii).

To prove the well-known fact (iv), let $l, r$ denote respectively the $K$-linear operators of left and right multiplication by $y$. Then, since $l$ and $r$ commute, we obtain

$$
e_{m}=(r-l)^{m}(x)=\sum_{i=0}^{m}(-1)^{i}\left(\begin{array}{c}
m \\
i
\end{array}\right) l i r^{m-i}(x)=\sum_{i=0}^{m}(-1)^{i}\left(\begin{array}{c}
m \\
i
\end{array}\right) y^{i} x y^{m-i} .
$$

Thus if $m=2 n-1$ and $R$ satisfies $y^{n}=0$, then every term in the sum on the right is zero.

Proposition 1.4. is a consequence of the following result.

Proposition 2.2. Let $R$ be a K-algebra.

(i) If $(R, \circ)$ satisfies a semigroup identity, then $(R, \cdot)$ satisfies the same identity.

(ii) If $(R, \circ)$ satisfies a semigroup identity then $(R, \circ)$ satisfies the corresponding reduced identity.

(iii) If $R$ is unital, then $(R, \circ) \cong(R, \cdot)$.

Proof. Let $S$ be the unital hull of $R$; that is, $S=R$ if $R$ is unital and $S=K 1 \oplus R$ if $R$ is nonunital. The map $\iota: r \rightarrow 1+r$ is an injective semigroup map from $(R, \circ)$ into $(S, \cdot)$ that is onto if (and only if) $R=S$. This proves (iii). The image under $\iota$ of an identity in $(R, \circ)$ is an identity in $(1+R, \cdot) \subseteq(S, \cdot)$. Only the bottom degree homogeneous component of this identity involves 1 and the other homogeneous components yield identities in $(R, \cdot)$. The highest degree component is precisely the original identity, yielding (i).

Assume that $u(x, y)=v(x, y)$ is an identity for $(R, \circ)$ of degree $n$. Write $u=a u^{\prime} b, v=a v^{\prime} b$, where $u^{\prime}=v^{\prime}$ is a reduced equation. We show that $u^{\prime}=v^{\prime}$ also holds in $(R, \circ)$. It suffices, by symmetry and by induction on the maximum length of $a$ and $b$, to prove this in the case when $a=x$ and $b$ is empty. The identity $x u^{\prime}(x, y)=x v^{\prime}(x, y)$ in $(R, \circ)$ is equivalent to the polynomial identity

$$
(1+x) u^{\prime}(1+x, 1+y)-(1+x) v^{\prime}(1+x, 1+y)=0
$$

in $R$. Let $m$ be an even integer with $m \geq n+1$. Then multiplying the last identity on the left by $1-x+x^{2}-\cdots+x^{m}$ yields the polynomial identity

$$
\left(1+x^{m+1}\right) u^{\prime}(1+x, 1+y)-\left(1+x^{m+1}\right) v^{\prime}(1+x, 1+y)=0 .
$$


Separating homogeneous components and using the fact that $x^{m+1}$ has higher $x$ degree than $u^{\prime}$ and $v^{\prime}$, we obtain the polynomial identity

$$
u^{\prime}(1+x, 1+y)-v^{\prime}(1+x, 1+y)=0
$$

in $R$, which is equivalent to $u^{\prime}=v^{\prime}$ holding in $(R, \circ)$. This proves (ii).

The following lemma is crucial to our main theorems and is best possible in view of Example 1.2. A simpler argument, as in [2], is available in characteristic zero. That argument fails in positive characteristic, where the situation is more delicate.

Lemma 2.3. Suppose that $R$ satisfies $y^{m} \alpha y^{k}=0$, where $\alpha=\sum_{i=0}^{n} \alpha_{i} y^{i} x y^{n-i}$.

(i) If $\alpha$ is right reduced, then $R$ satisfies $y^{m+n} e_{n} y^{k}=0$.

(ii) If $\alpha$ is left reduced, then $R$ satisfies $y^{m} e_{n} y^{n+k}=0$.

(iii) If $\alpha$ is reduced, then $R$ satisfies $y^{m} e_{3 n-1} y^{k}=0$.

Proof. By symmetry, the proof of (ii) is entirely analogous to that of (i). If the conclusions of (i) and (ii) hold, then the conclusion of (iii) follows from equation (1):

$$
y^{m} e_{3 n-1} y^{k}=y^{m} \sum_{i=0}^{2 n-1}(-1)^{i}\left(\begin{array}{c}
2 n-1 \\
i
\end{array}\right) y^{i} e_{n} y^{2 n-1-i} y^{k}=0 .
$$

Thus it suffices to prove the conclusion of (i).

First assume that $m=k=0$. Make the substitution $y \mapsto y(y+1)$. Expanding $\sum_{i=0}^{n} \alpha_{i} y^{i}(y+1)^{i} x y^{n-i}(y+1)^{n-i}=0$ by the binomial theorem and separating homogeneous components yields identities $v_{0}=0, \ldots, v_{n}=0$ for $R$, where $v_{r}$ is homogeneous of degree $n+r$ in $y$. We claim that

$$
\sum_{r=0}^{n}(-1)^{r} v_{r} y^{n-r}=\alpha_{n} y^{n} e_{n}
$$

To establish equation (2), it suffices to show that the coefficients of $y^{a} x y^{2 n-a}$ on each side are equal, whenever $0 \leq a \leq 2 n$.

First note that by equation (1), the coefficient of $y^{a} x y^{2 n-a}$ in $y^{n} e_{n}$ is $(-1)^{a-n}\left(\begin{array}{c}n \\ a-n\end{array}\right)$ if $a \geq n$ and 0 otherwise. With the usual convention on binomial coefficients, the expression $(-1)^{a-n}\left(\begin{array}{c}n \\ a-n\end{array}\right)$ is valid for all $a$. Using the same convention we may sum over all values of any index occurring.

Now we calculate the coefficient of $y^{a} x y^{2 n-a}$ in $v_{r} y^{n-r}$ or, what is the same, the coefficient of $y^{a} x y^{n+r-a}$ in $v_{r}$. The binomial theorem expansion above shows that the coefficient of $y^{s} x y^{t}$ is precisely $\sum_{i+j=n} \alpha_{i}\left(\begin{array}{c}i \\ s-i\end{array}\right)\left(\begin{array}{c}j \\ t-j\end{array}\right)$. Putting $s=a$ and $t=r+n-a$, we obtain the desired coefficient as $\sum_{i} \alpha_{i}\left(\begin{array}{c}i \\ a-i\end{array}\right)\left(\begin{array}{c}n-i \\ r-(a-i)\end{array}\right)$.

It follows that 


$$
\begin{aligned}
& \sum_{r}(-1)^{r} \sum_{i} \alpha_{i}\left(\begin{array}{c}
i \\
a-i
\end{array}\right)\left(\begin{array}{c}
n-i \\
r-(a-i)
\end{array}\right) \\
& =\sum_{i} \alpha_{i}\left(\begin{array}{c}
i \\
a-i
\end{array}\right) \sum_{r}(-1)^{r}\left(\begin{array}{c}
n-i \\
r-(a-i)
\end{array}\right) \\
& =\sum_{i} \alpha_{i}\left(\begin{array}{c}
i \\
a-i
\end{array}\right)(-1)^{a-i} \sum_{s}(-1)^{s}\left(\begin{array}{c}
n-i \\
s
\end{array}\right) \\
& =(-1)^{a-n}\left(\begin{array}{c}
n \\
a-n
\end{array}\right) \alpha_{n},
\end{aligned}
$$

since the inner sum has the value zero, unless $n-i=0$, and 1 otherwise. This proves (i) in the case $m=k=0$.

In the general case, where $m$ and $k$ are not necessarily zero, the substitution $y \mapsto y(y+1)$ into the original identity yields an identity

$$
\sum_{r+s+t \leq m+n+k} c_{r s t} y^{s}\left(y^{m} v_{r} y^{k}\right) y^{t}=0
$$

for some coefficients $c_{r s t} \in K$. For $0 \leq a \leq n$, consider the homogeneous component of (3) of degree $m+n+k+a$ in $y$. The only $v_{r}$ occurring have $r \leq a$ and the only term involving $v_{a}$ is precisely $y^{m} v_{a} y^{k}$. By induction on $a, y^{m} v_{r} y^{k}=0$ is an identity in $R$ for all $r<a$, and hence so is $y^{m} v_{a} y^{k}=0$. We may now proceed exactly as in the special case above and the conclusion follows.

2.1. Unital algebras. In case $R$ is unital, more information can be obtained. Note that $e_{n}(x, y)=x(\operatorname{ad} y)^{n}=x(\operatorname{ad}(y+1))^{n}=e_{n}(x, y+1)$. Thus by substituting $y \mapsto y+1$ into the result of (i) or (ii) in Lemma 2.3. and separating out the component of degree $n$ in $y$ we obtain $e_{n}=0$ in $R$.

In the rest of this subsection (which is not essential to the main results of the paper) we give a characterization (for unital $K$-algebras) of the Engel identities.

For each $m \geq 0$, let $W_{m}$ be the $K$-submodule of $K\langle x, y\rangle$ with basis all monomials $y^{i} x y^{j}$ such that $i+j=m$, and let $V_{n}=\sum_{m=0}^{n} W_{m}$ and $V=\sum_{n \geq 0} V_{n}$. Note that $W_{0}$ is spanned by the monomial $x$, and that, for $n \geq 1, e_{n}$ is a reduced element of $W_{n}$.

Define the difference operator $\Delta$ on $V$ by $\Delta \alpha(x, y)=\alpha(x, y+1)-\alpha(x, y)$. Note that $\Delta: V_{n} \rightarrow V_{n-1}$, and that the homogeneous component of degree $n-1$ in $y$ of $\Delta \alpha$ is simply the Hausdorff derivative $\partial \alpha / \partial y$ with respect to $y$ (that is, the unique $K$ derivation of $K\langle x, y\rangle$ sending $y$ to 1 and $x$ to 0 ).

Proposition 2.4. Let $R$ be a unital K-algebra, and $\alpha \in W_{n}$.

(i) $\Delta \alpha=0$ if and only if $\alpha$ is a scalar multiple of $e_{n}$.

(ii) If char $K=0$, then $\partial \alpha / \partial y=0$ if and only if $\alpha$ is a scalar multiple of $e_{n}$.

Proof. Given $\alpha(x, y)=\sum_{i=0}^{n} \alpha_{i} y^{i} x y^{n-i}$, expand $\alpha(x, y+1)$ by the binomial theorem. The coefficient of $y^{s} x y^{t}$ in $\alpha(x, y+1)$ is given by 


$$
\left[y^{s} x y^{t}\right]= \begin{cases}\sum_{i} \alpha_{i}\left(\begin{array}{l}
i \\
s
\end{array}\right)\left(\begin{array}{ll}
n-i \\
t
\end{array}\right) & (s+t<n), \\
0 & (s+t=n) .\end{cases}
$$

Now $\Delta \alpha=0$ if and only if the coefficients of all monomials $y^{i} x y^{j}$, for $i+j \leq n-1$, are zero. This gives a system of linear equations in the $n+1$ unknowns $\alpha_{0}, \ldots, \alpha_{n}$. We claim that the coefficient matrix $M$ has rank exactly $n$. Indeed, by equation (4), the submatrix of rows corresponding to the components of $x y^{m}, 0 \leq m \leq n-1$, has the form

$$
\left[\begin{array}{cccccc}
* & 1 & 0 & 0 & \cdots & 0 \\
* & * & 1 & 0 & \cdots & 0 \\
\vdots & \vdots & \vdots & \ddots & \vdots & 0 \\
* & * & \cdots & * & 1 & 0 \\
* & * & * & \cdots & * & 1
\end{array}\right]
$$

which shows that the rank is at least $n$. However the rank is not $n+1$ since, as observed above, the coefficient vector $\alpha_{i}=(-1)^{i}\left(\begin{array}{l}n \\ i\end{array}\right)$ of $e_{n}$ is in the kernel of $M$. This proves (i).

To prove (ii), it suffices to show that in characteristic zero, the submatrix of $M$ consisting of all rows corresponding to monomials $y^{s} x y^{t}$, with $s+t=n-1$, has rank $n$. By equation (4), this submatrix has the form

$$
\left[\begin{array}{cccccc}
n & 1 & 0 & 0 & \ldots & 0 \\
0 & n-1 & 2 & 0 & \ldots & 0 \\
0 & 0 & n-2 & 3 & \ldots & 0 \\
\vdots & \vdots & \vdots & \ddots & \ddots & 0 \\
0 & 0 & 0 & \ldots & 1 & n
\end{array}\right] .
$$

Since char $K=0$ the submatrix consisting of the first $n$ columns is nonsingular and (ii) follows.

3. Proofs of theorems. We first prove Theorem 1.1. The implication (ii) $\Rightarrow$ (iii) is obvious. Also (i) $\Rightarrow$ (ii) and (iii) $\Rightarrow$ (iv) follow from Proposition 2.2. and Proposition 2.1, respectively. By Lemma 2.3 with $m=k=0$, (iv) and (v) are equivalent. Suppose then that $R$ satisfies $e_{n}=0$. Let $x, y \in R$. By [14], the subalgebra $T$ of $R$ generated by $x$ and $y$ is Lie nilpotent of class $m$ depending on $n$ only. An easy induction on $m$ shows that $T$, and hence $R$, satisfies the Morse identity, in the circle sense, of degree $m$. Indeed,

$$
u_{m}-v_{m}=\left[u_{1}, v_{1}, v_{2}, \ldots, v_{m-1}\right]
$$

This proves (v) $\Rightarrow$ (vi). The last implication (vi) $\Rightarrow$ (i) is obvious.

We now prove Theorem 1.3. The implication (i) $\Rightarrow$ (ii) is obvious; (ii) $\Rightarrow$ (iii) follows from Proposition 2.1, and (iii) $\Rightarrow$ (iv) can be deduced from Lemma 2.3. If $K=p>0$ then (iv) $\Rightarrow$ (i), since by increasing $m$ if necessary we may assume that $m=p^{t}$, so that 


$$
y^{p^{t}} x y^{2 p^{t}}-y^{2 p^{t}} x y^{p^{t}}=y^{p^{t}} e_{p^{t}} y^{p^{t}}=0 \text {. }
$$

Finally, Theorem 1.5 follows from the quantitative form of Theorem 1.1 and [14, Theorem].

4. Comments. In an earlier version of this paper, we asked the following questions about an arbitrary ring $R$. These questions arose naturally from the work above, and the converses had been shown to hold in [14].

- If a ring $R$ satisfies a reduced semigroup identity, does $R$ necessarily satisfy an Engel identity?

- If a ring $R$ satisfies a reduced circle semigroup identity, does $R$ necessarily satisfy an Engel identity?

We are indebted to Ol'ga Paison for showing us that the answer to both is no. We now present her example.

Let $p$ be a prime, let $F$ be a field of order $p^{2}$ and let $R$ be the subring of $M_{2}(F)$ consisting of all elements of the form $a e_{11}+a^{p} e_{22}+b e_{12}$, for $a, b \in F$. Then $R$ does not satisfy any Engel identity. To see this, choose $a \in F$ with $a^{p} \neq a$. Let $x=a e_{11}+a^{p} e_{22}$ and $y=e_{12}$. Then, for sufficiently large even integers $s$, we have $\left[x^{p^{s}}, y\right]=\left(a-a^{p}\right) e_{12} \neq 0$. On the other hand, the only idempotents of $R$ are 0 and 1 , and so $R$ satisfies a reduced (circle) semigroup identity in view of the following result.

Proposition 4.1. Let $R$ be a finite ring. Then

(i) $(R, \cdot)$ and $(R, \circ)$ satisfy an identity of the form $x^{t} \equiv x^{2 t}$.

(ii) If all idempotents of $R$ are central, then $R$ satisfies a reduced semigroup identity.

Proof. The conclusion of part (i) is true for every finite semigroup $S$. First, every element of $S$ is periodic. Furthermore, every periodic element in a semigroup has some power which is an idempotent. To see this, note that for a fixed $x \in S$, $x^{m}=x^{m+a}$, for some $m, a>0$. This implies that, for all $n \geq 1$ and all $s \geq m$, $x^{s}=x^{s+n a}$. Choose $t_{x}$ such that $t_{x} \geq m$ and $a$ divides $t_{x}$. Then $\left(x^{t_{x}}\right)^{2}=x^{t_{x}}$. The desired global identity follows directly from this equation, since $S$ satisfies $x^{t} \equiv x^{2 t}$ with $t=\prod_{x \in S} t_{x}$.

Now by (i), there is some $t$ for which $x^{t}$ is an idempotent, for each $x \in R$. Thus if all idempotents of $R$ are central, $R$ satisfies the identity $x^{t} y \equiv y x^{t}$, yielding (ii).

In [2] it was shown (using arguments special to characteristic zero) that the $K$ algebra $R$ satisfies a partial linear identity if and only if the algebra of $2 \times 2$ upper triangular matrices over $K$ is not in the variety generated by $R$. Perhaps this is true in all characteristics.

ACKNOWLEDGEMENTS. The first author received support from NSF-EPSCoR in Alabama and the University of Alabama Research Advisory Committee. The second author is supported by a NZST Postdoctoral Fellowship. This work was done 
while the first author visited the Department of Mathematics at the University of Auckland, and their hospitality is gratefully acknowledged.

\section{REFERENCES}

1. S. I. Adian, The Burnside problem and identities in groups (Springer-Verlag, 1979).

2. I. Z. Golubchik and A. V. Mikhalev, On varieties of algebras with a semigroup identity, Vestnik Moskov. Univ. Ser. I Mat. Mekh. 37 no. 2 (1982), 8-11. 1035 .

3. J. Levitzki, On a problem of A. Kurosch, Bull. Amer. Math. Soc. 52 (1946), 1033-

4. J. Lewin and T. Lewin, Semigroup laws in varieties of solvable groups, Proc. Camb. Phil. Soc. 65 (1969), 1-9.

5. A. I. Mal'cev, Nilpotent semigroups, Ivanov. Gos. Ped. Inst. Uč;. Zap. Fiz.-Mat. Nauki 4 (1953), 107-111.

6. V. D. Mazurov and E. I. Khukhro (eds.), Unsolved problems in group theory: (The Kourovka notebook), 13th ed. (Russian Academy of Sciences Siberian Division, Institute of Mathematics, Novosibirsk, 1995).

7. M. Morse, Recurrent geodesics on a surface of negative curvature, Trans. Amer. Math. Soc. 22 (1921), 84-100.

8. B. H. Neumann and T. Taylor, Subsemigroups of nilpotent groups, Proc. Roy. Soc. Ser. A 274 (1963), 1-4.

9. A. Yu. Ol'shanskii and A. Storozhev, A group variety of relatively free groups, $J$. Austral. Math. Soc. Ser. A 60 (1996), 255-259.

10. A. Rhemtulla, private communication.

11. L. Rowen, Ring theory (Academic Press, 1988).

12. D. M. Riley, Algebras with collapsing monomials, Bull. London Math. Soc. 30 (1998), $521-528$

13. D. M. Riley and V. Tasić, Mal'cev nilpotent algebras, Arch. Math. (Basel) 72 (1999), $22-27$.

14. D. M. Riley and M. C. Wilson, Associate rings satisfying the Engel condition, Proc. Amer. Math. Soc. 127 no 4 (1999), 973-976.

15. D. M. Riley and M. C. Wilson, Group algebras and enveloping algebras with nonmatrix and semigroup identities, Comm. Algebra 27 no 7 (1999), 3545-3556.

16. A. Shalev, Combinatorial conditions in residually finite groups II, J. Algebra 157 (1993), 51-62.

17. A. Shalev, Finite p-groups, in Finite and locally finite groups (Istanbul, 1994), NATO Adv. Sci. Inst. Ser. C. Math. Phys. Sci. 471 (Kluwer Acad. Publ., Dordrecht, 1995), 401-450.

18. J. Shemple and A. Shalev, Combinatorial conditions in residually finite groups I, $J$. Algebra 157 (1993), 43-50.

19. E. I. Zel'manov, On the restricted Burnside problem, Siberian Math J. 30 (1990), $885-891$. 